\title{
Índice de accesibilidad para cibermedios mexicanos
}

\author{
Rafael Ochoa-Urrego \\ Facultad de Ciencias Políticas y Sociales de la Universidad Nacional Autónoma de México \\ Correo-e: rlochoau@unal.edu.co ORCID iD: https://orcid.org/0000-0003-1117-4877
}

Recibido: 23-11-2017; 2a versión: 03-05-2018; Aceptado: 18-02-2019.

Cómo citar este artículo/Citation: Ochoa-Urrego, R. (2019). Índice de accesibilidad para cibermedios mexicanos. Revista Española de Documentación Científica, 42 (3), e244. https://doi.org/10.3989/redc.2019.3.1541

Resumen: El presente artículo presenta la construcción del Índice de Accesibilidad para México MAI, el cual está orientado a medir los niveles de implementación de la WCAG 2.0 en los cibermedios mexicanos. Para conseguir este objetivo se evalúa el número de errores reportados por la herramienta TAW de 1531 cibermedios mexicanos. El MAI es construido utilizando AHP como método de ponderación, LST como método de normalización y la agregación geométrica. Se destaca el alto nivel de cumplimiento de la norma WCAG 2.0 lo que arroja un MAI promedio de $94.5 \%$. Este alto rendimiento es impulsado por los resultados sobresalientes en los principios de operabilidad y comprensión. Además, se encontró que el principio de robustez es el que presenta menores niveles de cumplimiento. Se destaca a www.contrasena.com.mx como el cibermedio con el mejor desempeño a la luz de los lineamientos.

Palabras clave: Accesibilidad web; TAW; cibermedios; periodismo digital; WCAG 2.0.

\section{Accessibility index for Mexican cybermedia}

Abstract: This article presents the construction of the Mexican Accessibility Index MAI, which is aimed to measure the levels of implementation of WCAG 2.0 standard in Mexican cybermedia. To achieve this, the number of errors reported by the TAW tool of 1531 Mexican cybermedia is evaluated. The MAI is constructed using AHP as a weighting method, LST as a normalization method and finally geometric aggregation. The high level of compliance with the WCAG 2.0 standard stands out, showing an average MAI of $94.5 \%$. This high performance is driven by outstanding results in the principles of operability and comprehension. In addition, it was found that lowest levels of compliance are related to the principle of robustness. It highlights www.contrasena.com. $\mathrm{mx}$ as the cybermedia with the best performance in light of the guidelines.

Keywords: Web Accesibility; TAW; cybermedia; online journalism; WCAG 2.0.

Copyright: (c) 2019 CSIC. Este es un artículo de acceso abierto distribuido bajo los términos de la licencia de uso y distribución Creative Commons Reconocimiento 4.0 Internacional (CC BY 4.0). 


\section{INTRODUCCIÓN}

Los países en desarrollo están en el proceso de consolidarse en la denominada sociedad del conocimiento. Para lograr esta consolidación es necesario conseguir niveles adecuados de apropiación de las Tecnologías de la Información y la Comunicación - TIC. Las TIC se caracterizan por buscar proveer un acceso universal a la web independientemente de la plataforma de software o hardware que el usuario utilice, de su cultura o de su ubicación geográfica (Roig-Vila y otros, 2014). Igualmente, este escenario genera procesos de comunicación que no dependen de la ubicación y no están condicionados a los límites físicos de los sistemas (Laudon y Guercio, 2009; Roig-Vila y otros, 2014). De manera consecuente, la apertura de los contenidos digitales propende por el acceso universal al conocimiento (Rodríguez y otros, 2017)

Infortunadamente, la gran cantidad de información y recursos disponibles en la red, se convierte en un arma de doble filo para personas con algún tipo de discapacidad (Roig-Vila y otros, 2014). Como respuesta a esta problemática, en los últimos años se han fortalecido los lineamientos para la creación de sitios web accesibles (Schiavone y Paternò, 2015). Como resultado al seguimiento de dichos lineamientos, se disminuyen las barreras de acceso para cualquier tipo de usuario (Urgilés y otros, 2017); generando nuevas y mejores experiencias (McHale, 2011). Como efecto colateral, las páginas web diseñadas con los estándares de accesibilidad incrementan la reputación del sitio y generan ganancias en confianza y lealtad de los usuarios (McHale, 2011).

Paralelamente, uno de los modelos de negocio que más aprovecha las potencialidades de la red y de la comunicación digital es el de los cibermedios (Navarro Zamora, 2011; Ochoa y Crovi, 2017). Este concepto agrupa a las organizaciones que generan, editan, distribuyen y comercializan información de corte noticioso a través de medios digitales (Burgueño, 2011; Cabrera González, 2009; Díaz Rodríguez y Marrero Santana, 2011; Gallardo, 2011; Krueger y otros, 2004; Palacios y Díaz Noci, 2009; Rodríguez-Martínezy otros, 2010; Said-Hung y Arcila-Calderón, 2011). Como consecuencia de la digitalización de los contenidos, los cibermedios modifican profundamente el modelo tradicional en términos del modelo de consumo y la creciente necesidad de participación por parte de las audiencias (Aragón Robles Linares, 2016).

La oferta de contenidos noticiosos digitales se ha incrementado en los últimos años. En México se reporta la aparición de cibermedios en 1995
(Crovi, 2016). En 1996 se identificaron 62 portales noticiosos (Aragón Robles Linares, 2016), 147 en 2002 (Crovi, 2016), 307 en 2008 (López Aguirre, 2010) y 1531 en 2017 (Ochoa y Crovi, 2017). Este crecimiento está acompañado por crecientes niveles de penetración de internet, llegando al 56\% para el 2017 (Reuters Institute, 2017). La combinación de una amplia gama de ofertas comunicativas y niveles de penetración crecientes han hecho que el $91 \%$ de los mexicanos utilicen algún medio digital como fuente de noticias, superando medios convencionales tales como la televisión $(65 \%)$, el periódico impreso (51\%) y la radio (33\%) (Reuters Institute, 2017).

Por otro lado, la población mexicana con algún tipo de discapacidad también se ha incrementado. En el 2010 se contabilizaron 5.739 .000 personas con discapacidad, representando el $5,15 \%$ de la población (INEGI, 2010). Para el 2014 la cifra ascendió a 7.100.000, que equivalen al $6 \%$ de la población (INEGI, 2016; Urgilés y otros, 2017). Con esta combinación de factores se hace necesario medir los niveles de implementación de los estándares de accesibilidad en cibermedios mexicanos; buscando concienciar a los medios de comunicación sobre la importancia de la accesibilidad (Roig-Vila y otros, 2014).

Para cumplir el objetivo central de este trabajo, se muestra el diseño del indicador compuesto "Índice de accesibilidad para México -MAI" construido usando AHP como método de ponderación, LST como método de normalización y la agregación geométrica. Para realizar la ponderación se consultaron 14 expertos mexicanos, obteniendo una tasa de consenso del 85,4\%. Además, se realizó la medición de los errores de accesibilidad mediante la utilización de TAW para 1531 cibermedios identificados en México.

El artículo se organiza de la siguiente manera. En la sección 2 se discuten las bases de la accesibilidad, el estándar WCAG y su evolución. En la tercera sección, se exponen los aspectos metodológicos que llevaron a la construcción del índice y su aplicación en los cibermedios mexicanos. En la sección 4, se muestra los resultados del estudio incluyendo el panorama mexicano en términos de la población con capacidades diferentes, las políticas de accesibilidad y los estudios de accesibilidad realizados anteriormente en entornos similares. Posteriormente, se aplica el MAI y se analiza estadísticamente los resultados obtenidos. Por último, los autores presentan las conclusiones más relevantes y posibles trabajos futuros. 


\section{ESTADO DEL ARTE}

En esta sección se discutirán las bases teóricas asociadas a la accesibilidad web y su medición a través de los estándares WCAG en sus distintas versiones. Igualmente, se discutirán algunos elementos que permiten conocer los abordajes dados en México a la aplicación de los conceptos de la accesibilidad en contenidos web.

\subsection{Revisión de la literatura}

La accesibilidad web pretende garantizar el acceso y correcto uso de un sitio web por parte de cualquier usuario (Urgilés y otros, 2017). En otras palabras, la accesibilidad busca que un usuario pueda hacer uso de las funcionalidades de un producto, servicio o recurso de una manera satisfactoria sin que influya la existencia de una capacidad diferente (Rodríguez y otros, 2017). Esto incluye no solamente poder navegar en un sitio web, sino también interactuar con otros usuarios y aportar contenidos (Casasola Balsells y otros, 2017). La importancia de la accesibilidad radica en la creciente digitalización de los contenidos, factor que actúa como una fuerte barrera de acceso para personas con capacidades distintas. Es así, que se hace necesaria la construcción de sitios web que cumplan con los principios básicos de la accesibilidad (Roig-Vila y otros, 2014)

Las normas de accesibilidad más aceptadas en el mundo son las desarrolladas por la Web Accessibility Initiative (WAI por sus siglas en inglés) del World Wide Web Consortium (W3C por sus siglas en inglés) (Rodríguez y otros, 2017; Sam-Anlas y Stable-Rodríguez, 2016). En 1999 el W3C lanza la primera versión de los lineamientos de accesibilidad para contenido web - WCAG por sus siglas en inglés (McHale, 2011). Para ese momento, las páginas web eran principalmente texto, por lo que los lineamientos de accesibilidad y su cumplimiento era relativamente simples (Murley, 2008). La primera versión de esta norma, o WCAG 1.0, define 14 elementos de comprobación para el diseño de contenidos accesibles para todos los usuarios los cuales no dependen del dispositivo de acceso (Schiavone y Paternò, 2015; World Wide Web Consortium, 1999).

Sin embargo, con la masificación de contenidos multimediales y la diversificación de opciones de plataformas de acceso se requería la creación de un estándar más preciso y claro para la implementación de los principios de la accesibilidad (Murley, 2008). Es por esto que en 2008 es lanzada la WCAG 2.0, la cual revisa de manera profunda los principios y elementos de comprobación planteados en la versión 1.0 (McHale, 2011; Murley, 2008; Schiavone y Paternò, 2015).

Esta nueva versión crea lineamientos para el diseño de contenidos accesibles involucrando un rango mayor de discapacidades. La nueva versión incluye discapacidades visuales, auditivas, motoras, de habla, cognitivas, de aprendizaje y neuronales. Así mismo, involucra el diseño de contenidos para personas que tienen cambios en sus capacidades como resultado del envejecimiento (World Wide Web Consortium, 2008).

WCAG 2.0 es una norma de tres capas. La primera capa está dedicada a definir los principios básicos para el diseño de contenidos. La segunda, define los lineamientos que detallan cada uno de los principios y determinan los objetivos principales que se deben seguir al momento de diseñar un contenido accesible. Y la tercera capa, define los criterios de éxito para cada uno de los lineamientos. Estos criterios se dividen en tres niveles $A, A A$ y AAA (Al-Khalifay otros, 2017; Rodríguez y otros, 2017; World Wide Web Consortium, 2008). El resumen de estas tres capas se muestra en la figura 1.

Figura 1. Componentes de WCAG 2.0

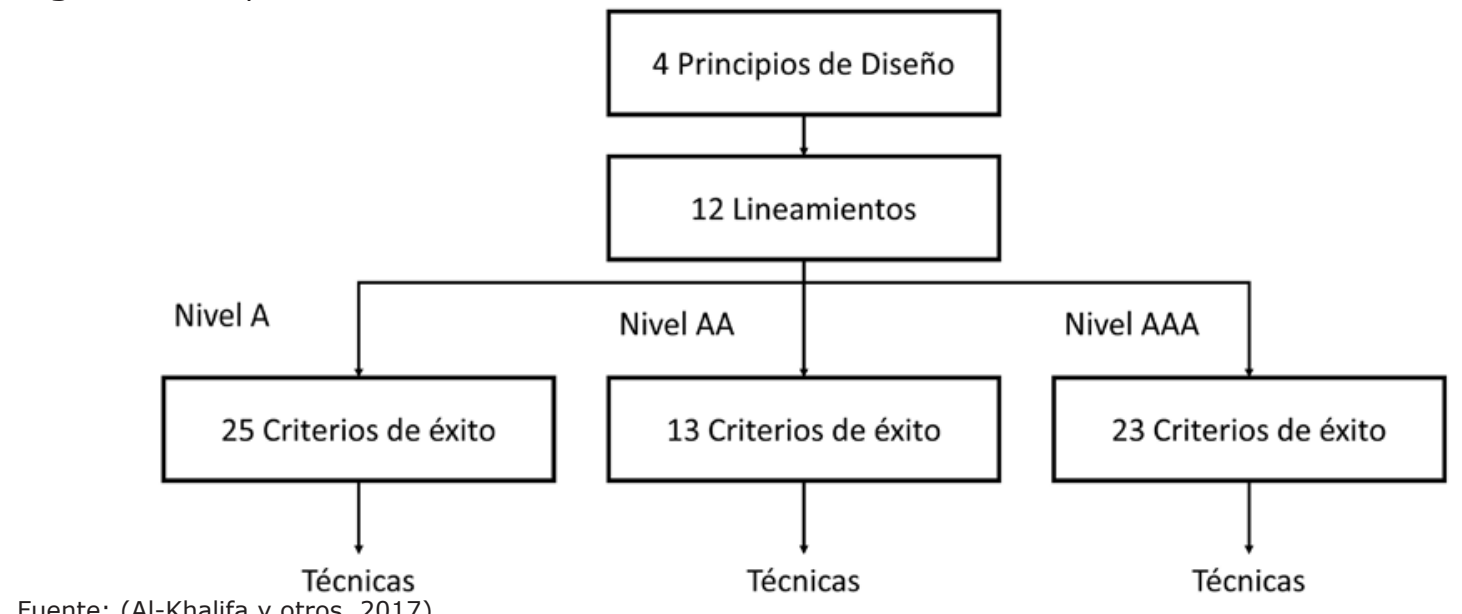


La base de la WCAG 2.0 está definida en los tres principios básicos para el diseño de contenidos. Comenzando por el principio de percepción, el cual determina que la información y los componentes de la interfaz no pueden ir más allá del entendimiento del usuario. El segundo principio o principio de operación determina que la interfaz no debe contener acciones que el usuario no pueda ejecutar. Por su parte, el principio de comprensión dispone que la información no puede ser invisible para todos los sentidos del usuario; además, establece que el contenido debe ser entendible sin importar las modificaciones que le imponga la tecnología de asistencia. Por último, el principio de robustez establece que el contenido debe permanecer accesible así los usuarios y las tecnologías de acceso evolucionen (Roig-Vila y otros, 2014; World Wide Web Consortium, 2017).

Adicionalmente, la $\mathrm{W} 3 \mathrm{C}$ ha definido los métodos de evaluación, WCAG - EM por sus siglas en inglés, donde se establecen los procedimientos de evaluación del cumplimiento de WCAG 2.0, incluyendo procesos de autoevaluación y de evaluación externa (Rodríguez y otros, 2017). Dentro de estos procesos de evaluación se contemplan 4 tipos. En primera instancia se consideran las herramientas de evaluación de la accesibilidad web, WEAT por sus siglas en inglés, las cuales son herramientas de software diseñadas para evaluar automáticamente el cumplimiento de los requerimientos de la norma. Estas herramientas son de fácil y rápida ejecución (Al-Khalifa y otros, 2017). Sin embargo, estas herramientas tienen la limitante de no poder captar la percepción ni la satisfacción del usuario (Al-Khalifa y otros, 2017; Kaur y Dani, 2017).

Adicionalmente, se contempla la evaluación por parte de expertos en accesibilidad. Con este tipo de evaluación se obtienen resultados más precisos, pero son más costosos en tiempo y recursos (AlKhalifa y otros, 2017). Igualmente, existe un alto componente de subjetividad e interpretación por parte de los expertos, disminuyendo su efectividad (Kaur y Dani, 2017).

Como últimas dos opciones de evaluación se encuentran las pruebas de los sitios por parte de los usuarios y encuestas con los desarrolladores de contenidos y plataformas. En el primer caso, este tipo de encuestas recogen la evaluación de la experiencia del usuario. Sin embargo, representan altos costos en la preparación de la prueba y elección de la muestra, actividad que puede condicionar los resultados. En el segundo caso, las encuestas con diseñadores están orientadas a identificar las razones por las cuales se presentan los problemas de accesibilidad (Al-Khalifa y otros, 2017).

\subsection{Discapacidad y accesibilidad web en México}

Antes de entrar a analizar el MAI es necesario conocer el contexto mexicano en dos puntos principales: la composición de la población que presenta algún tipo de discapacidad y el marco regulatorio de la accesibilidad en el país.

En primera instancia, en México se entiende por población en discapacidad aquellas personas que declaran no poder realizar o tener dificultades graves para realizar actividades básicas tales como ver, escuchar, caminar, recordar, cuidar de sí mismo o comunicarse (INEGI, 2016). A nivel mundial, el $10 \%$ de la población es identificada con algún tipo de discapacidad. En Estados Unidos esta población representa el 18,7\%, mientras que en la comunidad europea es un $16 \%$ (Schiavone y Paternò, 2015).

Como se estableció previamente, para el 2014 el $6 \%$, de la población mexicana tiene algún tipo de discapacidad. Asimismo, se estableció que la población mayor (60 años y más) es en la que se presentan mayores niveles de condiciones asociadas a la discapacidad. Además, se encontró que la población femenina con alguna discapacidad es mayor a la población masculina (3.8 millones de mujeres frente a 3.3 millones de varones) (INEGI, 2016).

Analizando el tipo de discapacidades, el $64.1 \%$ de la población con capacidades diferentes en México tiene problemas para caminar, por lo que las TIC se convierten en una importante herramienta de interacción para esta población. Por otro lado, el $58.4 \%$ de la población tiene algún tipo de discapacidad visual, mientras que el $33.5 \%$ tienen problemas de audición y el $18 \%$ tiene inconvenientes para comunicarse (INEGI, 2016). Es precisamente en esta población en la que el cumplimiento de los principios de accesibilidad se hace importante, ya que el diseño de contenidos y sistemas de información accesibles les permitiría acceder de manera masiva al mundo digital.

En consecuencia, el 13 de diciembre de 2006, las Naciones Unidas aprueban la convención sobre los derechos de las personas con discapacidad. Entre otras cosas, se define que la accesibilidad es uno de los 8 principios generales de dicha convención y se exhorta a los gobiernos miembros a incluir las leyes y regulaciones necesarias para "promover el acceso de las personas con discapacidad a los nuevos sistemas y tecnologías de la información y las comunicaciones, incluida Internet" (Naciones Unidas, 2006).

Por esta razón, muchos gobiernos crean leyes y regulaciones que buscan crear ambientes favorables para la implementación de los principios de la 
accesibilidad (Schiavone y Paternò, 2015). El movimiento inició en la década de 1990 en Estados Unidos (Murley, 2008), seguido por los países de la Comunidad Europea en la década del 2000 (Schiavone y Paternò, 2015). El primer país latinoamericano en presentar una legislación en el tema fue Brasil en 2004, seguido por Chile en 2006. En este momento el $55 \%$ de los países latinoamericanos cuentan con algún tipo de reglamentación relacionada con la accesibilidad (Urgilés y otros, 2017). En la Figura 2 se muestran algunos de los hitos importantes en la aparición de reglamentaciones relacionadas con la accesibilidad en el mundo.

Por su parte, México reglamenta la accesibilidad en el Acuerdo "por el que se establecen las Disposiciones generales de accesibilidad Web que deben observar las dependencias y entidades de la Administración Pública Federal y las empresas productivas del Estado", el 3 de diciembre de 2015. En este acuerdo se establecen las definiciones básicas relacionadas con la implementación de los principios de accesibilidad. Para tal fin, el Gobierno mexicano asume las definiciones propuestas por la WCAG 2.0 y propone como objetivo de cumplimiento el nivel AA (Secretaría de la Función Pública México, 2015).

Existen múltiples estudios que analizan la accesibilidad en distintos contextos. Uno de estos es el Web Assesment Index, el cual recoge ítems relativos a la accesibilidad, la rapidez, la navegabilidad y la calidad de los contenidos para 84 sitios de distintos países europeos (Piñeiro-Naval y otros, 2017). Por otro lado, Sam-Anlas y StableRodríguez realizan una evaluación de la accesibilidad de los portales del Estado en Perú (Sam-Anlas y Stable-Rodríguez, 2016). Los anteriores autores proponen un porcentaje de éxito en la implementación de 14 indicadores incluidos en la WCAG 2.0. Sin embargo, dicho porcentaje se realiza bajo una ponderación ad hoc lo cual no permite conocer realmente la importancia de cada una de las valoraciones realizadas. Un ejercicio similar es realizado por Casasola Balsells y otros (2017) quienes proponen una puntuación global de accesibilidad al promediar los resultados obtenidos por los sitios web de las universidades andaluzas en tres pruebas: el observatorio de accesibilidad del Ministerio de Hacienda Publica de España, una herramienta automática y una prueba manual. Una de las debilidades encontradas en esta propuesta, es el hecho de realizar el análisis solamente a nivel AA. Además, realiza una ponderación por pesos iguales de las tres pruebas, sin considerar la valoración de los indicadores internos.

Igualmente, Laitano (2015) realiza la evaluación de la accesibilidad en las universidades públicas en Argentina. En este caso se analiza el número promedio de universidades que incumplen con alguno de los principios generales de la WCAG. Como aporte interesante, la autora analiza cuál sería el grupo de personas con capacidades diferentes que serían más beneficiados por los distintos sitios web analizados.

A pesar de la posición manifiesta a favor de la implementación de la accesibilidad en México, hay pocos estudios que permitan establecer el cumplimiento de los principios establecidos por la WCAG 2.0. En 2008, Figueroa-Saavedra (2008) realiza una comparación de los niveles de implementación

Figura 2. Línea de tiempo de las reglamentaciones en accesibilidad

E.E.U.U.

American with Disabilities

Act

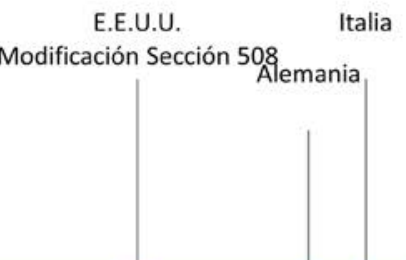

Francia

Puerto Rico

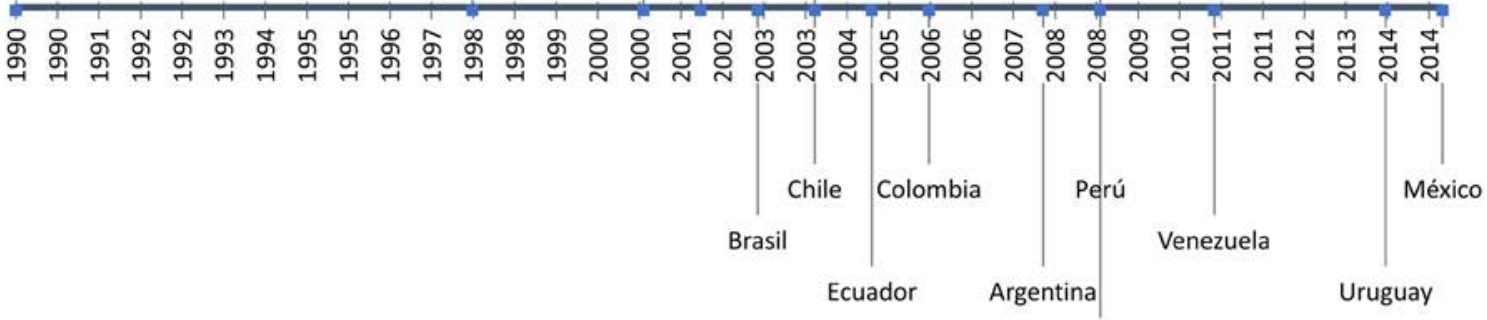

Bolivia 
de la accesibilidad en 16 entidades de salud del ámbito federal. Posteriormente, Morales y otros realiza, el comparativo de algunos sitios web de universidades mexicanas usando WCAG-EM (Morales Montelongo y otros, 2015).

En lo relacionado con los cibermedios mexicanos Said-Hung y Arcila (Said-Hung y Arcila-Calderón, 2011) realizaron la comparación de varios medios en América Latina, comparación en la que se incluyó al El Universal. Recientemente, Aragón (2016) compara cuatro cibermedios del Estado de Sonora. Estos dos estudios recopilan el número de errores que presentan los sitios web de los cibermedios y están orientados por la propuesta de medición de Rodríguez-Martínez y otros (2012). A pesar de los interesantes hallazgos de los estudios mencionados, su alcance no permite establecer de manera completa los niveles de cumplimiento de las normas de accesibilidad en el país, por lo que se hace necesario construir una plataforma metodológica que permita tener el alcance nacional.

\section{DISEÑO DE LA INVESTIGACIÓN}

El presente trabajo se desarrolló en dos etapas. La primera, consistió en la realización del inventario de cibermedios en México. Para esto se consolidaron diferentes listados de cibermedios existentes en el país. Entre los listados consultados se destacan el padrón de medios impresos de la Secretaría de Gobernación de México, el Catálogo Nacional De Medios Impresos e Internet 2014, desarrollado por el Instituto Federal Electoral, y el estudio realizado por López Aguirre (2010), entre otros. Esta información se complementó con búsquedas avanzadas conformadas por las palabras clave "Noticias", "Periódico", "Diario", "Revista", "Radio", "Emisora" y los nombres de los estados de la república mexicana. Como resultado se identificaron 1531 cibermedios.

Para realizar la medición de la accesibilidad de los cibermedios mexicanos identificados, se procedió a identificar las WEAT más pertinentes. En este sentido, se acudió al listado realizado por la WAI, disponible en https://www.w3.org/WAI/ER/tools/, en el cual se encuentran más de 93 herramientas relacionadas. Desafortunadamente, muchas de estas herramientas no se encuentran operativas, y otras tantas solo evalúan WGAC 1.0 (Schiavone y Paternò, 2015). Una de las herramientas más utilizadas y referenciadas en la literatura es TAW. Este es un software diseñado por la Fundación CTIC, oficina de la W3C en España, que ofrece análisis automáticos con una aproximación determinística y reportes estadísticos gráficos y fácilmente comprensibles (Roig-Vila y otros, 2014; Schiavone y Paternò, 2015). TAW incluye el análisis para las versiones 1.0 y 2.0 de WCAG (Rodríguez y otros, 2017), siendo la herramienta que evalúa de manera más completa las normas (Casasola Balsells y otros, 2017; Vigo y otros, 2013). Adicionalmente, TAW es la herramienta con mayor efectividad y menor número de falsos positivos en ambientes móviles (Kaur y Dani, 2017). Por estas razones se seleccionó TAW como la herramienta para realizar el análisis de los cibermedios mexicanos.

A la totalidad de los cibermedios identificados se le aplicó las pruebas TAW mediante la herramienta www.tawdis.net. En un momento inicial se consideró complementar las mediciones automáticas con mediciones manuales. Sin embargo, esta opción se descartó debido a la complejidad que tendría la tarea de analizar los 1531 cibermedios seleccionados para el estudio.

Se evaluó WCAG 2.0 con un nivel de análisis AAA y considerando la evaluación de HTML y CSS. Si bien es cierto que la reglamentación mexicana estipula el nivel de análisis AA como el objetivo, esto aplica para páginas web de entidades estatales o gubernamentales. Para el caso de los cibermedios, donde el componente multimedial es uno de los de mayor importancia, se hace necesario evaluar el nivel AAA el cual, entre otros elementos, incluye estándares de accesibilidad sobre audio y video (Moreno y otros, 2008).

Se consideraron como variables de análisis el número de errores reportados en los cuatro principios básicos: comprensión, operación, percepción y robustez. Todas las pruebas se aplicaron el 12 de septiembre de 2017. Luego de aplicar las pruebas se excluyeron 76 cibermedios. Estas exclusiones se realizaron ya sea porque el cibermedio desapareció entre el momento de la identificación y la aplicación de las pruebas o porque debido a la configuración de los sitios web no fue posible obtener resultados.

Como segunda etapa, se construyó el Índice de Accesibilidad para México (MAI) utilizando los métodos planteados por la OCDE para la construcción de índices compuestos (Nardo y otros, 2005; OCDE, 2008). La selección de métodos realizada para la construcción del índice se relaciona en la Tabla I.

Tabla I. Métodos para la construcción del índice compuesto

\begin{tabular}{ll}
\multicolumn{1}{c}{ Etapa } & \multicolumn{1}{c}{ Método } \\
\hline Normalización & Linear Scaling Technique - LST \\
Ponderación & Analytic Hierarchy Process - AHP \\
Agregación & Geométrica \\
\hline
\end{tabular}


En la etapa de ponderación, se aplicaron las matrices de comparación definidas por Saaty (Saaty, 2004, 2008; Saaty y Vargas, 2012) para AHP. Se diseñó una matriz de comparación, la cual fue consultada por 14 expertos en cibermedios en México. El listado de los expertos consultados para el estudio se encuentra en la tabla II. Tal como lo plantea la metodología AHP, las matrices de comparación cuantifican la percepción que tienen los expertos sobre la importancia relativa que tiene cada uno de los criterios. En otras palabras, cada experto, desde su campo de acción como investigador o ciberperiodista, pondera la importancia que tiene cada uno de los tipos de errores seleccionados para el presente análisis.

Tabla II. Expertos consultados en la fase de ponderación

\begin{tabular}{ll}
\hline Elemento & Selección \\
\hline Elba Díaz Cerveró & Universidad Panamericana \\
José Luis López Aguirre & Universidad Panamericana \\
Claudia Alicia Lerma Noriega & Tecnológico de Monterrey \\
Marco Lara Klahr & $\begin{array}{l}\text { Universidad Nacional } \\
\text { Autónoma de México }\end{array}$ \\
Rosalía Orozco & Universidad de Guadalajara \\
Tania Lucía Cobos & $\begin{array}{l}\text { Universitat Autònoma de } \\
\text { Barcelona }\end{array}$ \\
David Ramírez Plascencia & Universidad de Guadalajara \\
Juan S. Larrosa-Fuentes & Temple University \\
Elva Araceli Fabián González & Universidad de Guadalajara \\
Janny Amaya Trujillo & Universidad de Guadalajara \\
Gerardo Albarrán de Alba & Saladeprensa.org \\
Fidel Romero & Universidad de Guadalajara \\
Dolores Aragón Robles Linares & Universidad de Sonora \\
José Luis Ulloa Luna & Universidad de Guadalajara \\
María Elena Gutiérrez Rentería & Universidad Panamericana \\
\hline
\end{tabular}

Luego de eliminar los juicios con CR superiores al $10 \%$ quedaron 11 juicios válidos. El grado de conceso obtenido fue del $85,4 \%$. La matriz de ponderación resultante tiene $G C I=0.02$ y $C R=0,6 \%$. Con estos resultados se considera la ponderación como válida. Como resultado de la ponderación el peso asignado a los errores de percepción fue de $19.2 \%$, operación $26.4 \%$, comprensión $33.6 \%$ y robustez $20.8 \%$.

Como parte del método seleccionado para la construcción del índice compuesto, se realizó la combinación de técnicas expresada en la tabla III.
Como último paso, se calculó el MAI obtenido por cada uno de los cibermedios y se construyó el ranking respectivo. Como parte del análisis se comparó el índice de accesibilidad promedio de cada uno de los estados de la república mexicana con el porcentaje de población con algún tipo de discapacidad. Esta comparación se realizó con la idea de tener un panorama de la conciencia que tiene los cibermedios de dicha población. Adicionalmente, se analizan los resultados obtenidos en cada uno de los principios de la WCAG mediante variables estadísticas descriptivas.

Tabla III. Estadísticos descriptivos básicos

\begin{tabular}{ll}
\hline \multicolumn{1}{c}{ Elemento } & Selección \\
\hline Escala de Medición & Balanceada \\
Método de Priorización & $R G M M$ \\
Medición inconsistencia individual & $C R$ \\
Valor máximo de aceptación & $C R \leq 10 \%$ \\
Tipo de Agregación & $A I J$ \\
Método de Agregación & $R G M M$ \\
Medición de consistencia matriz agrupada & $G C I$ \\
\hline
\end{tabular}

RGMM Método de media geométrica por filas, $C R$ índice de consistencia, AIJ Agregación de juicios individuales, GCI índice de consistencia geométrica

\section{4. ÍNDICE DE ACCESIBILIDAD PARA MÉXICO - MAI}

El índice de accesibilidad para México - MAI agrupa los resultados en términos de errores en la medición TAW para los cibermedios identificados. Con este fin se realizó la medición de dichos errores para los 1462 cibermedios, igualmente que el cálculo del MAI para cada uno de estos. Los estadísticos descriptivos básicos de las mediciones se muestran en la Tabla IV.

Como primer elemento a resaltar es el valor de las modas para las cuatro variables. Estos valores bajos dan cuenta de un buen comportamiento en términos del cumplimiento de la norma. Por otro lado, se observa que todos los tipos de errores presentan coeficientes de variación superiores a 1 , lo que implica un alto nivel de heterogeneidad de las variables. Igualmente, los valores muy altos de curtosis y los valores positivos de asimetría llevan a concluir que los valores muestran una distribución Leptocúrtica con una alta concentración a la izquierda de la media y no se asimilan a una distribución normal. 
Tabla IV. Estadísticos descriptivos básicos

\begin{tabular}{|c|c|c|c|c|}
\hline & Perc. & Oper. & Compr. & Rob. \\
\hline Máximo & 774 & 1838 & 118 & 214 \\
\hline Mínimo & 0 & 0 & 0 & 0 \\
\hline Moda & 1 & 2 & 1 & 1 \\
\hline Promedio & 53.30 & 52.74 & 3.16 & 25.68 \\
\hline $\boldsymbol{\sigma}$ & 66.66 & 77.52 & 5.94 & 35.69 \\
\hline Coeficiente de variación & 1.25 & 1.46 & 1.88 & 1,38 \\
\hline Rango Intercuartílico & 60.75 & 57.75 & 2 & 23 \\
\hline Asimetría & 3.91 & 10.44 & 9.29 & 2.89 \\
\hline Curtosis & 27.84 & 204.3 & 136.45 & 9.43 \\
\hline
\end{tabular}

Perc. Perceptibles, Oper. Operables, Compr. Comprensión, Rob. Robustez

Adicionalmente, se observa que el peor comportamiento obtenido por los cibermedios está en los errores de operación, comportamiento que preocupa debido a que este ítem es valorado como el segundo en importancia por los expertos. Sin embargo, y como compensación, los cibermedios presentaron un muy buen comportamiento en los ítems de errores de comprensión, variable ponderada como la más importante por los expertos.

Al realizar el cálculo del MAI se obtuvo que el cibermedio www.contrasena.com.mx de Sonora tiene un cumplimiento del $99.961 \%$. Mientras que los medios www.307rivieramaya.com, www. diariojudio.com, www.informaciondelonuevo.com y www.antorcha.net presentan el peor comportamiento con índices de cero. Este resultado se explica por el hecho de que los medios mencionados presentan el mayor número de errores en alguno de los tipos de errores de accesibilidad usados para el análisis. En la Tabla $\mathrm{V}$ se muestran los diez cibermedios con mejor y peor comportamiento en el indicador compuesto.

Seguidamente se realizó el análisis del MAI por cada uno de los estados de la república. Como complemento, se comparó el MAI con el porcentaje de población con capacidades diferentes en cada uno de los estados. Los resultados se muestran en la Figura 3.

En primera instancia, se resalta el buen comportamiento de los cibermedios de los estados de Nuevo León, Aguascalientes, Baja California Sur y Durango, los cuales obtuvieron MAI de 96\%.

En la comparación del MAI vs el porcentaje de población con capacidades diferentes, se encontró que los seis estados que presentan un menor MAI (Zacatecas, Colima, Yucatán, Querétaro, Sinaloa y
Baja California) son aquellos que presentan un mayor promedio de población con algún tipo de discapacidad. Esto hace que sea necesario implementar acciones para mejorar el cumplimiento de la norma y así poder llegar de una manera más acertada a las poblaciones con capacidades diferentes de estas regiones. Igualmente, es válido resaltar los resultados obtenidos por los cibermedios de los estados de Nayarit y Durango. En estos estados se presentan los mayores niveles de población con discapacidad y los cibermedios obtienen resultados sobresalientes, $95.7 \%$ y $96 \%$ respectivamente.

De manera general se encontró que los cibermedios mexicanos tiene buenos niveles de implementación de la norma WCAG 2.0 con un promedio de $94.5 \%$ lo que demuestra importantes avances en la cobertura de la población con capacidades diferentes del país. Estos resultados están impulsados principalmente por el muy buen desempeño en términos de los errores de comprensión y operación los cuales presentan un promedio de cumplimiento del $97.32 \%$ y $97.13 \%$ respectivamente. Sin embargo, es necesario realizar acciones prontas para mejorar el desempeño en términos de los errores de robustez, ya que los cibermedios tan solo alcanzan un $88 \%$ de cumplimiento.

Por último, y como complemento a los datos anteriores, se analizaron los resultados obtenidos en el MAI por los cibermedios mexicanos con mayor nivel de visibilidad y popularidad. Con este fin se seleccionaron los 20 primeros cibermedios en el ranking de Alexa. Dicho ranking mide, entre otras cosas, el número de enlaces entrantes y salientes, la visibilidad en los buscadores web y el número de visitantes y usuarios únicos. Los medios identificados mediante este ranking y su MAI se especifican en la tabla VI. 
Tabla V. Cibermedios con mejores y peores resultados

\begin{tabular}{|c|c|c|c|}
\hline \multicolumn{4}{|c|}{ Cibermedios con mejores resultados } \\
\hline Posición en el MAI & Sitio & Ubicación & Puntaje \\
\hline 1 & contrasena.com.mx & Sonora & $99,961 \%$ \\
\hline 2 & laotral.com.mx & Ciudad de México & $99,932 \%$ \\
\hline 3 & elprovincial.mx & Veracruz & $99,863 \%$ \\
\hline 4 & cronicadexalapa.com & Veracruz & $99,863 \%$ \\
\hline 5 & dqr.com.mx & Quintana Roo & $99,863 \%$ \\
\hline 6 & poresto.net & Nacional & $99,863 \%$ \\
\hline 7 & asisucedegto.mx & Guanajuato & $99,863 \%$ \\
\hline 8 & alertachiapas.com & Chiapas & $99,863 \%$ \\
\hline 9 & radioenciso.com & Aguascalientes & $99,835 \%$ \\
\hline 10 & tribunalibrenoticias.com & Veracruz & $99,824 \%$ \\
\hline \multicolumn{4}{|c|}{ Cibermedios con peores resultados } \\
\hline Posición en el MAI & Sitio & Ubicación & Puntaje \\
\hline 1462 & 307rivieramaya.com & Ciudad de México & $0,000 \%$ \\
\hline 1461 & diariojudio.com & Nacional & $0,000 \%$ \\
\hline 1460 & informaciondelonuevo.com & Yucatán & $0,000 \%$ \\
\hline 1459 & antorcha.net & Nacional & $0,000 \%$ \\
\hline 1458 & imagenzac.com.mx & Zacatecas & $40,269 \%$ \\
\hline 1457 & frontera.info & Baja California & $42,077 \%$ \\
\hline 1456 & elimparcial.com & Sonora & $43,709 \%$ \\
\hline 1455 & sintesis.mx & Puebla & $47,508 \%$ \\
\hline 1454 & aztecadeportes.com & Ciudad de México & $48,046 \%$ \\
\hline 1453 & sumedico.com & Ciudad de México & $48,051 \%$ \\
\hline
\end{tabular}

Figura 3. MAI vs población con discapacidad por estado

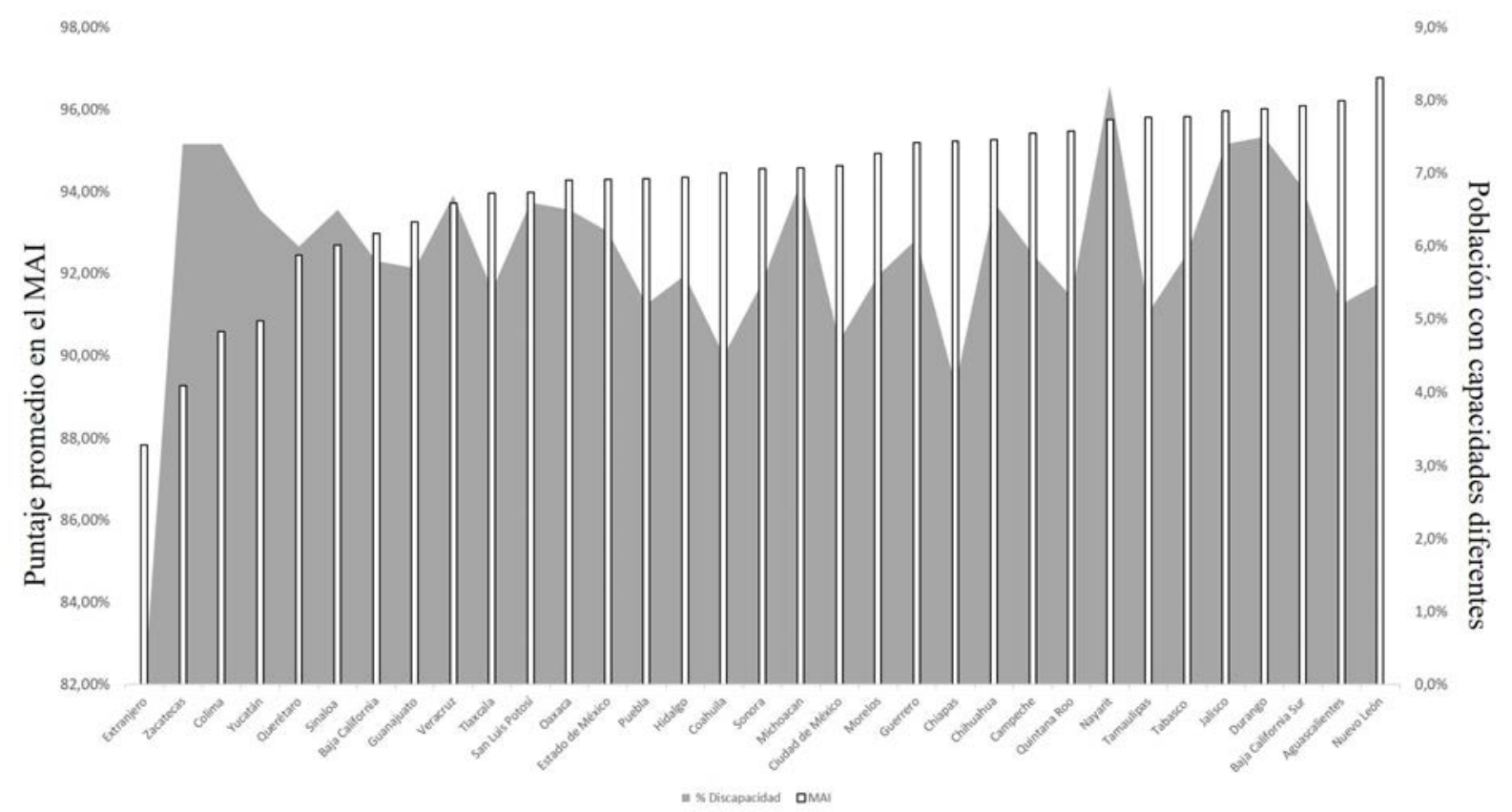


Tabla VI. Resultados del MAI para los principales Cibermedios en México

\begin{tabular}{|c|c|c|c|c|}
\hline Ranking Alexa & Sitio & Ubicación & Posición MAI & Puntaje \\
\hline 23 & debate.com.mx & Sinaloa & 1341 & $85,74 \%$ \\
\hline 27 & jornada.com.mx & Ciudad de México & 838 & $95,45 \%$ \\
\hline 32 & sopitas.com & Nacional & 1379 & $82,83 \%$ \\
\hline 46 & eluniversal.com.mx & Ciudad de México & 1335 & $86,10 \%$ \\
\hline 93 & milenio.com & Ciudad de México & 1144 & $92,18 \%$ \\
\hline 114 & lacasadelcurioso.com & Nacional & 276 & $98,60 \%$ \\
\hline 120 & tecreview.itesm.mx & Ciudad de México & 875 & $95,19 \%$ \\
\hline 128 & eslamoda.com & Nacional & 761 & $95,90 \%$ \\
\hline 129 & excelsior.com.mx & Ciudad de México & 588 & $96,88 \%$ \\
\hline 136 & diario.mx & Chihuahua & 1039 & $93,46 \%$ \\
\hline 149 & televisa.com & Ciudad de México & 319 & $98,30 \%$ \\
\hline 168 & proceso.com.mx & Ciudad de México & 1179 & $91,75 \%$ \\
\hline 175 & elfinanciero.com.mx & Ciudad de México & 541 & $97,11 \%$ \\
\hline 178 & mediotiempo.com & Ciudad de México & 1254 & $89,73 \%$ \\
\hline 179 & Sdpnoticias.com & Ciudad de México & 933 & $94,66 \%$ \\
\hline 184 & radio.ipn.mx & Ciudad de México & 34 & $99,66 \%$ \\
\hline 185 & aristeguinoticias.com & Ciudad de México & 1283 & $88,80 \%$ \\
\hline 188 & somosmexicanos.mx & Nacional & 493 & $97,33 \%$ \\
\hline 199 & laafoka.com & Nacional & 176 & $99,11 \%$ \\
\hline
\end{tabular}

En primera instancia, se puede concluir que no existe una relación directa entre la posición en el ranking de Alexa y el desempeño obtenido por los cibermedios en términos del MAI. Contrario a lo esperado, los principales medios del país no se encuentran en las primeras posiciones en lo relacionado con el cumplimiento de las normas de accesibilidad web. Aspecto que es preocupante, teniendo en cuenta que son estos medios los que tienen una audiencia mayor y por lo tanto no estarían dando cobertura suficiente a la población con algún tipo de capacidad diferente. En este grupo de 20 cibermedios, se destaca el resultado obtenido por www. radio.ipn. $\mathrm{mx}^{1}$, medio que obtiene la mejor posición en el MAI para esta muestra.

Adicionalmente, se observa que los tres cibermedios mejor ubicados según el indicador MAI (radio. ipn.mx, laafoka.com y lacasadelcurioso.com) son cibermedios alternativos y nativos digitales. Esto da pie a concluir que estos medios, debido a la comprensión que tienen de las herramientas digitales propias del mundo de internet, además de tener una amplia audiencia, han interiorizado de una mejor manera las normas de accesibilidad.

\section{CONCLUSIONES Y RECOMENDACIONES}

Es necesario un esfuerzo considerable para lograr que los cibermedios mexicanos cumplan de manera satisfactoria los lineamientos de accesibilidad. Este esfuerzo se hace aún más importante si se tiene en cuenta que su cumplimiento es un esfuerzo de carácter voluntario y en ocasiones invisible para la mayoría de la población.

Igualmente, se hace evidente que la población con algún tipo de discapacidad en México viene en crecimiento, por lo que se hace necesario que se redoblen esfuerzos para brindarle a esta población posibilidades de acceso acordes a sus habilidades.

Los resultados obtenidos muestran que a nivel país se tienen MAI satisfactorios, por lo que se reconoce el esfuerzo realizado por los cibermedios en su cumplimiento. Sin embargo, aún queda pendiente revisar el cumplimiento de la norma en el principio de robustez. Conclusión que coincide con lo encontrado por Aragón Robles Linares (2016); quien afirma que el $43 \%$ de los errores de accesibilidad encontrados en los medios analizados del estado de Sonora corresponden a errores de robustez. 
Así mismo, se encontró que en algunos estados los cibermedios obtienen niveles MAI comparativamente menores frente a la población con capacidades diferentes. Por esta razón se hace necesario crear conciencia en las organizaciones de dichos estados para que incrementen la importancia dada a la implementación de los lineamientos de accesibilidad.

Igualmente, se encontró que los cibermedios con mayores niveles de visibilidad y popularidad en la red, no son los que tienen el mejor desempeño en términos del cumplimiento de las normas de accesibilidad; siendo los cibermedios independientes y nativos digitales los que mejor desempeño presentan, lo que implica un mayor nivel de comprensión de las necesidades propias del entorno digital. Este comportamiento coincide con los hallazgos obtenidos por SaidHung y Arcila-Calderón (2011) quienes encontraron que los medios digitales de circulación nacional de los principales países de América Latina son aquellos que presentan un peor desempeño en términos de la implementación de técnicas de accesibilidad. Esto hace pensar que, a pesar del avance en el desarrollo de los contenidos y técnicas de publicación de contenidos web, la accesibilidad sigue sin ser un elemento relevante para los medios de amplia difusión.

Por tales razones, se hace urgente que el sector de los cibermedios en México, gane conciencia sobre la importancia de la implementación de este tipo de lineamientos con el fin de abrir la puerta a las personas con capacidades distintas a los contenidos y la información que ofrecen. Esto generaría una ganancia en doble parte: los lectores

\section{NOTAS}

1. En el momento de la publicación del artículo este dominio fue desactivado y migrado a https://tunein.

\section{REFERENCIAS}

Al-Khalifa, H. S.; Baazeem, I.; Alamer, R. (2017). Revisiting the accessibility of Saudi Arabia government websites. Universal Access in the Information Society, vol.16(4), 1027-1039. https://doi.org/10.1007/ s10209-016-0495-7

Aragón Robles Linares, D. (2016). Panorama y desafío en el periodismo on-line de Sonora : El Imparcial, Uniradio Noticias, Expreso y El Diario de Sonora. Revista de La Asociación Española de Investigación de La Comunicación, vol.3(5), 16-23.

Burgueño, J. M. (2011). La metamorfosis de la prensa. Hacia un nuevo modelo periodístico. TELOS - Cuadernos de Comunicación e Innovación, 86, 1-12.

Cabrera González, M. Á. (2009). El diseño de la prensa digital española en el contexto de la convergencia tecnológica. La identidad visual del ciberperiodismo. Revista Latina de Comunicación Social, 64, 766-786. https:// doi.org/10.4185/RLCS-64-2009-860-766-786 con capacidades distintas podrían tener acceso a la información, al mismo tiempo que los cibermedios empiezan a generar valor a partir de un segmento poco considerado en la actualidad.

\section{LIMITACIONES Y TRABAJO FUTURO}

Se reconoce que el estudio tiene, como principal limitación, la utilización de una única herramienta de evaluación como fuente de información. Esto puede condicionar los resultados al desempeño de TAW frente a la evaluación automática. Por lo tanto, podría plantearse una próxima investigación contemplando el uso de más de una herramienta.

Igualmente, se reconoce que la evaluación automática, elección realizada para aumentar la cobertura del estudio, puede imponer cierto sesgo al trabajo, por lo que se considera importante que se incluya posteriormente una evaluación manual de sitios.

Por último, se considera que sería muy interesante poder realizar paralelos con otros países de la región y así poder establecer un panorama regional del cumplimiento de la norma.

\section{AGRADECIMIENTOS}

Los resultados de este trabajo forman parte del proyecto "Construcción del ranking de cibermedios en México" financiado por el programa de becas para estancias posdoctorales en la UNAM, de la Dirección de Asuntos del Personal Académico - DGAPA de la Universidad Nacional Autónoma de México.

com/radio/Radio-IPN-El-politcnico-en-radio-957s10687/?lang=es

Casasola Balsells, L. A.; Guerra González, J. C.L; Casasola Balsells, M. A.; Pérez Chamorro, V. A. (2017). La accesibilidad de los portales web de las universidades públicas andaluzas. Revista Española de Documentación Científica, 40(2), e169. https://doi.org/10.3989/ redc. 2017.2 .1372

Crovi, D. (2016). México. en R. Salaverría (coord), Ciberperiodismo en Iberoamerica pp. 241-254. Madrid, España: Fundación Telefónica - Editorial Ariel S.A.

Díaz Rodríguez, E.; Marrero Santana, L. (2011). Nuevas fronteras para la participación en red. Ciberperiodismo y telefonía móvil en América Latina. Revista TELOS Cuadernos de Comunicación e Innovación, 87, 1-13.

Figeroa-Saavedra, M. (2008). Accesibilidad a la información digital para las personas con discapacidad en los servicios de información de ámbito federal en salud de México. Altepepaktli Salud de La Comunidad, 4(8), 3-13. 
Gallardo, F. (2011). En la era de los contenidos Digitales. Nuevos modelos de negocio para la prensa. TELOS Cuadernos de Comunicación e Innovación, 87, 1-11.

INEGI. (2010). Las personas con discapacidad en México, una visión al 2010. Disponible en: https://www.ipomex. org.mx/recursos/ipo/files_ipo3/2018/44257/4/ b202c98e9a2106f4c0f427b64f542c93.pdf [Fecha de Consulta: $10 / 10 / 2017]$

INEGI. (2016). La discapacidad en México, datos al 2014. Disponible en: http://internet.contenidos. inegi.org. $\mathrm{mx} /$ contenidos/productos/prod_serv/ contenidos/espanol/bvinegi/productos/nueva_estruc/702825090203.pdf [Fecha de Consulta: 10/10/2017].

Kaur, A.; Dani, D. (2017). Comparing and evaluating the effectiveness of mobile Web adequacy evaluation tools. Universal Access in the Information Society, 16(2), 411-424. https://doi.org/10.1007/s10209$016-0466-z$

Krueger, C. C.; Beek, K. Van Der; Swatman, P. M. C. (2004). New And Emerging Business Models For Online News : A Survey of 10 European Countries. En BLED 2004 Proceedings, pp. 1-16.

Laitano, M. I. (2015). Accesibilidad web en el espacio universitario público argentino. Revista Española de Documentación Científica, 38(1), e079. https://doi. org/10.3989/redc.2015.1.1136

Laudon, K.; Guercio, C. (2009). e-commerce: negocios, tecnología, sociedad (4a ed.). Ciudad de México: Pearson Education.

López Aguirre, J. L. (2010). Diagnóstico de la prensa digital Mexicana. Revista Mexicana de Comunicación.

McHale, N. (2011). An Introduction to Web Accessibility, Web Standards, and Web Standards Makers. Journal of Web Librarianship, 5(2), 152-160. https://doi.org/10. $1080 / 19322909.2011 .572434$

Morales Montelongo, J. G.; Neville Calixto, F.; Guiérrez Díaz de León, L. A. (2015). La estrategia de la Universidad de Guadalajara en la implementación de accesibilidad web en los portales universitarios. En Quinta Conferencia de Directores de Tecnología de Información, TICAL 2015 Gestión de las TICs para la Investigación y la Colaboración, Viña del Mar, 6-8 de julio.

Moreno, L.; Martínez, P.; Ruiz-Mezcua, B. (2008). Disability Standards for Multimedia on the Web. IEEE Multimedia, 15(4), 52-54. https://doi.org/10.1109/ MMUL. 2008.85

Murley, D. (2008). Web Site Accessibility. 100 Law Library Journal, vol.401(408), 8-23.

Naciones Unidas. (2006). Convención sobre los derechos de las personas con discapacidad y protocolo facultativo. https://www.un.org/disabilities/documents/convention/convoptprot-s.pdf

Nardo, M.; Saisana, M.; Saltelli, A.; Tarantola, S. (2005). Tools for composite indicators building. Institute for the Protection and Security of the Citizen Econometrics and Statistical Support to Antifraud Unit I-21020 Ispra.

Navarro Zamora, L. (2011). Orígenes del ciberperiodismo. Correspondencias y Análisis, 1, 61-76. https:// doi.org/10.24265/cian.2011.n1.04

OCDE. (2008). Handbook on Constructing Composite Indicators. Methodology and user guide.

Ochoa, R. L.; Crovi, D. (2017). Caracterización de los cibermedios mexicanos: una visión desde la difusión de innovaciones. en Congreso Internacional de Innovación y tendencias en la ingeniería - CONIITI 2017. Bogotá.

Palacios, M.; Díaz Noci, J. (2009). Online journalism : research methods. Bilbao: Servicio Editorial de la Universidad del País Vasco.

Piñeiro-Naval, V.; Igartua, J. J.; Marañón, F. (2017). El diseño de las sedes web municipales de España. Una propuesta metodológica para su análisis. Revista Española de Documentación Científica, 40(1), e164. https://doi.org/10.3989/redc.2017.1.1368

Reuters Institute. (2017). Digital News Report 2017. https://bit.ly/2tSeHbL

Rodríguez-Martínez, R.; Codina, L.; Pedraza-Jiménez, R. (2010). Cibermedios y web 2.0: modelo de análisis y resultados de aplicación. El Profesional de La Informacion, 19(1), 35-44. https://doi.org/10.3145/epi.2010.ene.05

Rodríguez-Martínez, R.; Codina, L. ; Pedraza-Jiménez, R. (2012). Indicadores para la evaluación de la calidad en cibermedios: análisis de la interacción y de la adopción de la Web 2.0. Revista Española de Documentación Científica, 35(1), 61-93. https://doi.org/10.3989/ redc.2012.1.858

Rodríguez, G.; Pérez, J.; Cueva, S.; Torres, R. (2017). A framework for improving web accessibility and usability of Open Course Ware sites. Computers and Education, 109, 197-215. https://doi.org/10.1016/j.compedu.2017.02.013

Roig-Vila, R.; Ferrández, S.; Ferri-Miralles, I. (2014). Assessment of web content accessibility levels in Spanish official online education environments. International Education Studies, 7(6), 31-45. https://doi. org/10.5539/ies.v7n6p31

Saaty, T. L. (2004). Decision making - the Analytic Hierarchy and Network Processes (AHP/ANP). Journal of Systems Science and Systems Engineering, 13(1), 1-35. https://doi.org/10.1007/s11518-006-0151-5

Saaty, T. L. (2008). Decision making with the analytic hierarchy process. Services Sciences, 1(1), 83-98. https://doi.org/10.1504/IJSSCI.2008.017590

Saaty, T. L.; Vargas, L. G. (2012). Models, methods, concepts \& applications of the Analitic Hierarchy Process ( $2^{\mathrm{a}}$ ed.). Springer New York Heidelberg Dordrecht London. https://doi.org/10.1007/978-1-4614-3597-6

Said-Hung, E.; Arcila-Calderón, C. (2011). Los cibermedios en América Latina y la Web 2.0. Comunicar: Re- 
vista Científica de Educomunicación, 19(37), 125-131. https://doi.org/10.3916/C37-2011-03-04

Sam-Anlas, C. A.; Stable-Rodríguez, Y. (2016). Evaluación de la accesibilidad web de los portales del Estado en Perú. Revista Española de Documentación Científica, 39(1), e120. https://doi.org/10.3989/ redc.2016.1.1213

Schiavone, A. G.; Paternò, F. (2015). An extensible environment for guideline-based accessibility evaluation of dynamic Web applications. Universal Access in the Information Society, 14(1), 111-132. https://doi. org/10.1007/s10209-014-0399-3

Secretaría de la Función Pública México. (2015). Acuerdo por el que se establecen las Disposiciones generales de accesibilidad Web que deben observar las dependencias y entidades de la Administración Pública Federal y las empresas productivas del Estado. Disponible en: https:// bit.ly/2WCNyMR [Fecha de Consulta: 18/11/2017]

Urgilés, C.; Célleri-Pacheco, J.; Maza-Córdova, J. (2017). Accesibilidad web : un desafío para los desarrollado- res de América Latina. Conference Proceedings UT$M A C H, 1(1), 61-64$.

Vigo, M.; Brown, J.; Conway, V. (2013). Benchmarking web accessibility evaluation tools: Measuring the harm of sole reliance on automated tests. Proceedings of the 10th International Cross-Disciplinary Conference on Web Accessibility - W4A '13, pp. 1-10. https://doi. org/10.1145/2461121.2461124

World Wide Web Consortium. (1999). Web Content Accessibility Guidelines 1.0. Disponible en: https://www.w3.org/ TR/WAI-WEBCONTENT/ [Fecha de Consulta: 1/6/2017]

World Wide Web Consortium. (2008). Web Content Accessibility Guidelines (WCAG) 2.0. Disponible en: https://www.w3.org/TR/WCAG20/ [Fecha de Consulta: $1 / 6 / 2017]$

World Wide Web Consortium. (2017). How to Meet WCAG 2.0. Disponible en: https://www.w3.org/WAI/ WCAG20/quickref/ [Fecha de Consulta: 1/6/2017]

\section{ANEXO}

\section{Glosario}

\begin{tabular}{ll}
\hline Acrónimo & Significado \\
\hline AHP & Análisis de Proceso Jerárquico \\
LST & Transformación Lineal Estándar \\
MAI & Índice de Accesibilidad para México \\
TAW & Test of Accesibility Web \\
TIC & Tecnologías de la Información y la Comunicación \\
W3C & World Wide Web Consortium \\
WAI & Iniciativa para la Accesibilidad Web \\
WCAG & Lineamientos de Accesibilidad para Contenido Web \\
WCAG - EM & Métodos de evaluación para los Lineamientos de Accesibilidad para Contenido Web \\
WEAT & Herramientas de evaluación de la accesibilidad Web \\
\hline
\end{tabular}

